\title{
Formation Mechanism of the Dominant Kinks in GaP Nanowires Grown in an In- situ (S)TEM Gas Cell Holder
}

\author{
David Krug, Maximilian Widemann, Felix Gruber, Andreas Beyer and Kerstin Volz
}

Faculty of Physics and Materials Sciences Center, Philipps-Universität Marburg, Marburg, Hessen, Germany

Nanowires (NW), especially those consisting of III/V semiconductor materials, are used for many technical applications, like photonic devices as for example LEDs, photo detectors, lasers and solar cells [1]. To develop such devices an exact understanding of the growth behavior such as growth rate, growth direction or kinking mechanisms is required. A widely used fabrication process of these materials is metal organic vapor phase epitaxy (MOVPE), which is capable of layer growth as well as of nanowire formation. In-situ (scanning) transmission electron microscopy ((S)TEM) allows to investigate dynamic processes, which occur during growth. Gas environmental cells and heating holders enable to supply gases while heating the sample so that conditions comparable to those during the MOVPE-process can be realized in any microscope [2].

To this end, a commercially available Protochips Inc. in-situ system has been modified. In order to allow the usage of toxic and pyrophoric gases, like the precursor gases used in MOVPE growth, a gas mixing system, an appropriate gas monitoring system, as well as a gas scrubbing system have been added [3]. A

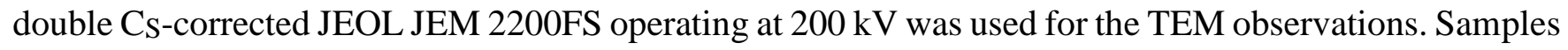
were prepared by depositing Au nanoparticles (NP) from a suspension droplet onto a micro electro mechanical system (MEMS) chip. Growth took place through vapor liquid solid (VLS) growth [4] catalyzed by the Au NP. Precursors used for GaP NW VLS growth were tertiarybutylphosphine and trimethylgallium at partial pressures between $10^{-1}$ and $10^{-2} \mathrm{hPa}$ and a V/III ratio of around 10 . Additionally, $\mathrm{N}_{2}$ was used as carrier gas at pressures of $400 \mathrm{hPa}$. Growth was performed at a temperature of $400{ }^{\circ} \mathrm{C}$. Preparation for post growth investigations took place using a JEOL JIB-4601F Multi-BeamSystem.

Our results show straight growing NW, which change their growth direction after random distances, producing kinking angles [Fig.1]. Statistics of these angles show different predominance angles [Fig.2] which are related to different formation mechanisms.

Growth in gas environmental cells comes along with several challenges, like the single tilt capabilities of the gas cell holder or the diffuse signals from the $\mathrm{SiN}$ windows. This technical framework often makes it impossible to achieve atomic resolved images of the sample, during growth and also in post growth investigations. Because of that a method was developed to lift the grown nanowires from the MEMS chip to a conventional TEM grid. This preparation enabled TEM measurements without diffuse background signal and with double tilt capability. These investigations give insights into the kinking mechanisms of the NW. We present the developed nanowire lifting procedure and the mechanism of kink formation investigated by $(\mathrm{S}) \mathrm{TEM}$. 


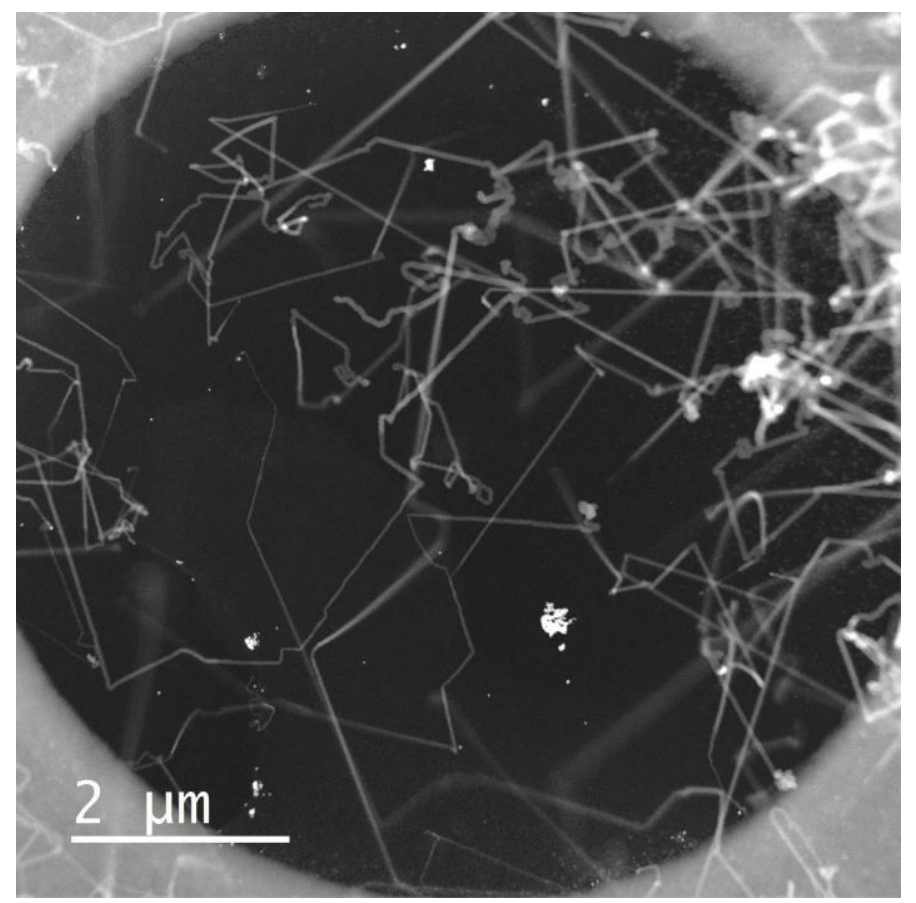

Figure 1. (S)TEM image of kinked GaP-NW on SiN window of the MEMS chip

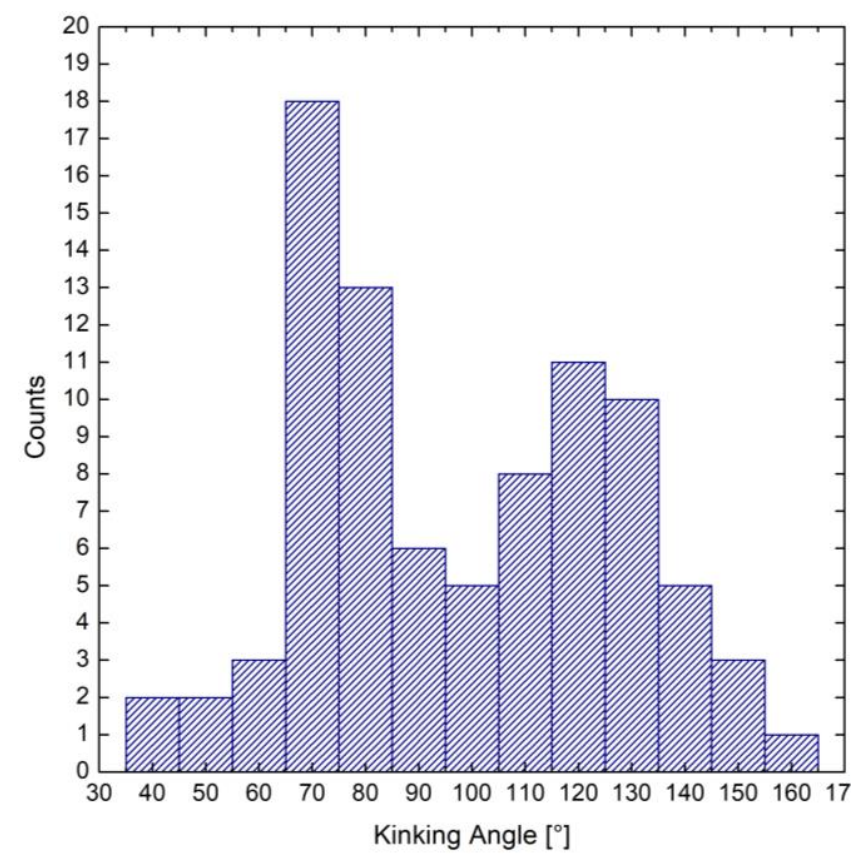

Figure 2. Histogram of the angular distribution of kinks in GaP-NW

\section{References}

[1] R. Yan, et al., Nature Photonics 3 (2009), pp. 569-576.

[2] L. F. Allard, et al., Microscopy and Microanalysis 18.4 (2012), pp. 656-666.

[3] R. Straubinger, et al., Microscopy and Microanalysis 23.4 (2017), pp. 751-757.

[4] R. S. Wagner, W. C. Ellis, Applied Physics Letters 4 (1964), pp. 89. 